\title{
Usage of PEN as self-vetoing structural material in the LEGEND experiment
}

\author{
Luis Manzanillas, ${ }^{a, *}$ Y. Efremenko, ${ }^{c}$ M. Febbraro, ${ }^{b}$ F. Fischer, ${ }^{a}$ M. Guitart \\ Corominas, ${ }^{a}$ B. Hackett, ${ }^{c}$ C. Hayward, ${ }^{d}$ R. Hodák, ${ }^{e}$ B. Majorovits, ${ }^{a}$ \\ D. Muenstermann, ${ }^{d}$ M. Pohl, ${ }^{f}$ R. Rouhana, ${ }^{f}$ D. Radford, ${ }^{b}$ E. Rukhadze, ${ }^{e}$ I. Schilling, ${ }^{f}$ \\ O. Schulz, ${ }^{a}$ I. Štekl, ${ }^{e}$ M. Stommel ${ }^{g}$ and J. Weingarten ${ }^{f}$ \\ ${ }^{a}$ Max-Planck-Institut für Physik, 80805 Munich, Germany \\ ${ }^{b}$ Oak Ridge National Laboratory, Oak Ridge, Tennessee 37830 \\ ${ }^{c}$ Department of Physics and Astronomy, University of Tennessee, Knoxville, Tennessee 37916 \\ ${ }^{d}$ Department of Physics, Lancaster University, Lancaster \\ ${ }^{e}$ Institute of Experimental and Applied Physics, Czech Technical University in Prague, CZ-11000 Prague, \\ Czech Republic \\ ${ }^{f}$ Technische Universität Dortmund, Dortmund \\ ${ }^{g}$ Leibniz-Institut für Polymerforschung Dresden e.V., 01069 Dresden, Germany \\ E-mail: manzanil@mpp.mpg.de
}

PEN is an industrial polyester plastic interesting for the physics community as a new type of wavelength shifting plastic scintillator. Recently, PEN structures with good radio-purity and attenuation length have been successfully produced using the injection molding technology. This opens the possibility for usage of PEN as optically active structural components in low-background experiments such as the LEGEND-200 experiment. In this contribution the ongoing R\&D on PEN is outlined with focus on the evaluation of the optical properties of PEN.

*** The European Physical Society Conference on High Energy Physics (EPS-HEP2021), ***

*** 26-30 July $2021 * * *$

*** Online conference, jointly organized by Universität Hamburg and the research center DESY ***

\footnotetext{
*Speaker
} 


\section{Introduction}

Rare event physics experiments as neutrinoless double decay $(0 v \beta \beta)$ searches demand ultra low backgrounds. Hence, ultrapure materials are required for the detectors and in all the structural components. In addition, active shields are also required to improve the discrimination of external backgrounds. Among the different approaches looking for $0 v \beta \beta$, Germanium $(\mathrm{Ge})$ detectors enriched in ${ }^{76} \mathrm{Ge}$ are one of the most promising technologies as demonstrated by the GERDA experiment [1]. The next generation of ${ }^{76} \mathrm{Ge} 0 v \beta \beta$ experiments LEGEND-200 and LEGEND-1000 will pursue the GERDA approach. To reach the background goals of LEGEND-1000, which is aiming for the deployment of a ton of enriched material, new innovations are needed. The structural materials around the detectors are an important source of backgrounds. These materials do not contribute to the veto systems and above all absorb light that can be used to identify backgrounds. Thus, all the inactive structural materials around the detectors have to be minimized in order to optimize light collection and decrease the backgrounds originated in the near vicinity of the detectors.

In recent years and as part of the LEGEND R\&D program, the PEN consortium has developed radio-pure active structural components. This innovation will allow to replace inactive materials around the detectors with radio-pure components made of poly(ethylene naphthalate) known as PEN. Being a scintillating material with wavelength shifting capabilities, PEN improves the light collection of the active veto system, resulting in a higher background rejection efficiency.

\subsection{The LEGEND experiment}

The LEGEND collaboration aims to develop a phased, ${ }^{76} \mathrm{Ge}$-based $\left(Q_{\beta \beta}=2039.06 \mathrm{keV}\right)$ doublebeta decay experimental program with discovery potential at a half-life beyond $10^{28}$ years. This half-life translates in less than one decay per year per ton of material. Thus 10 ton-years of data are required to get a few counts. Moreover, an excellent signal-to-background ratio in order to get statistical significance is needed. Hence, the two experimental challenges are having a very low background event rate and the best possible energy resolution.

The Ge-based technology provides mayor advantages. First, the best energy resolution on the field of $\sigma(\mathrm{E}) / \mathrm{E}<0.1 \%$ at $Q_{\beta \beta}$ has been demonstrated. Second, the source of $0 v \beta \beta$ and the detector are the same instrument and therefore a high detection efficiency and topology information are possible. Third, high-purity material can be used to build the detectors with no intrinsic background. Following the GERDA approach, Liquid Argon (LAr) serves to cool down the Ge detectors and as active shielding. The LAr scintillation light is readout using wavelength shifting fibers and SiPMs as shown in Figure 1. Currently the commissioning of LEGEND-200 in an upgraded GERDA infrastructure is ongoing.

\section{The PEN scintillator}

Polyethylene naphthalate is a commercially available polyester with a yield strength higher than copper at cryogenic temperatures. Almost any arbitrary shape with excellent transparency can be produced using the injection molding technology. In addition, PEN scintillates in the blue regime, with an emission spectrum peaking around $440 \mathrm{~nm}$, which is ideal for standard photo-sensors. PEN 

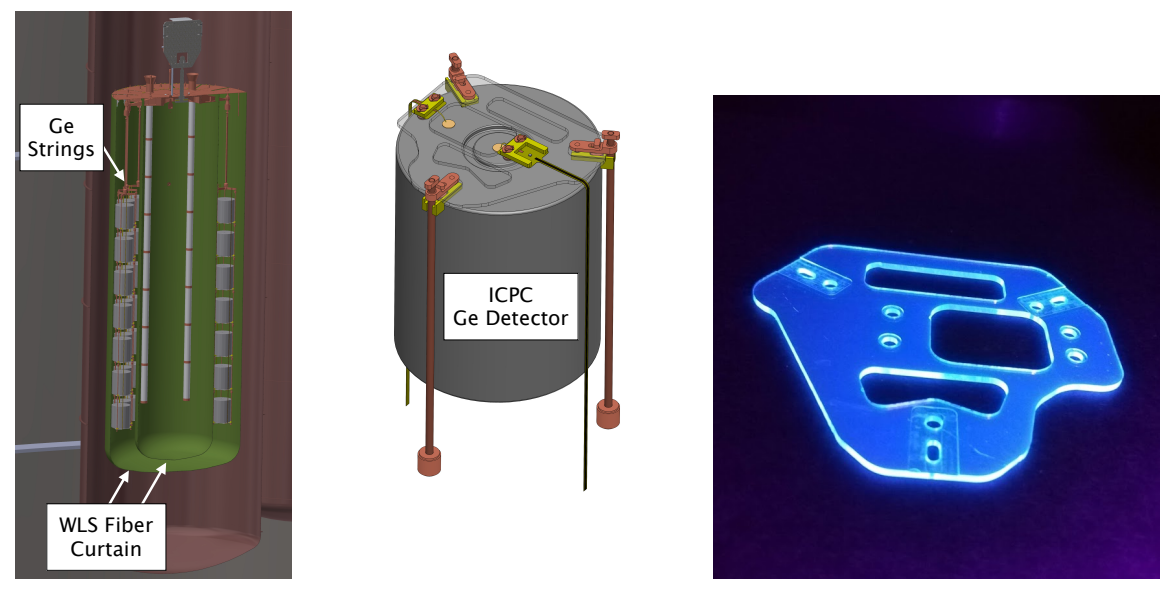

Figure 1: Infrastructure required to deploy the Ge detectors in the LEGEND-1000 experiment. The Ge detectors are mounted in strings using low mass structures. The base plate to place the front-end electronics and high voltage will be made of scintillating PEN (Right).

also has wavelength shifting properties with an efficiency of around 50\% of commonly used TPB [3]. Recently it has been identified as a potential self-vetoing structural material in low background physics experiments [2, 4].

As part of the LEGEND R\&D program, PEN discs with a thickness of $1.5 \mathrm{~mm}$ were produced under clean room conditions. Before the molding process the commercial raw material underwent a cleaning process in order to remove surface radio-impurities. The molded discs were then used to machine base holders to mount the Ge detectors in the LEGEND-200 experiment (see Figure 1) . The radiopurity achieved in this production corresponds in less than $1 \mu \mathrm{Bq}$ per holder, meeting the LEGEND-200 requirements. Finally, the usage of these PEN holders was validated during the post-GERDA tests, where Ge detectors were deployed with PEN holders. R\&D is ongoing for further application of PEN in the LEGEND-1000 experiment.

\subsection{PEN optical characterization}

Since PEN is a new plastic scintillator being used as an active structural material for the first time in the LEGEND experiment, most of its optical properties are unknown. In order to measure and characterize the optical properties of the PEN production for usage in the LEGEND-200 experiment, several setups have been mounted at the Max Planck Institute for Physics in Munich. Thus, the PEN light attenuation was studied using samples of $74 \times 20 \times 1.5 \mathrm{~mm}^{3}$. These samples were excited at different positions and the light was collected using PMTs coupled to both ends of the samples. The decrease of the light output as function of the distance from the excitation point to the PMTs is shown in Figure 2 (Left). The PEN emission spectrum was also measured using a spectrometer and exciting the sample at different points. From these two measurements and using simulations, a bulk absorption of $\sim 45 \mathrm{~mm}$ at $450 \mathrm{~nm}$ was found. The light output was measured using PEN samples of $30 \times 30 \times 1.7 \mathrm{~mm}^{3}$ coupled two 5 PMTs. The samples were then excited using electrons from a ${ }^{207} \mathrm{Bi}$ source. Standard plastic scintillator samples of the same dimensions were used for direct comparison as shown in Figure 2 (Right). From these results it was found that 
the PEN light yield is at least 3500 photons per MeV. Nevertheless the attenuation of PEN being not negligible, this value can be taken as a lower limit.
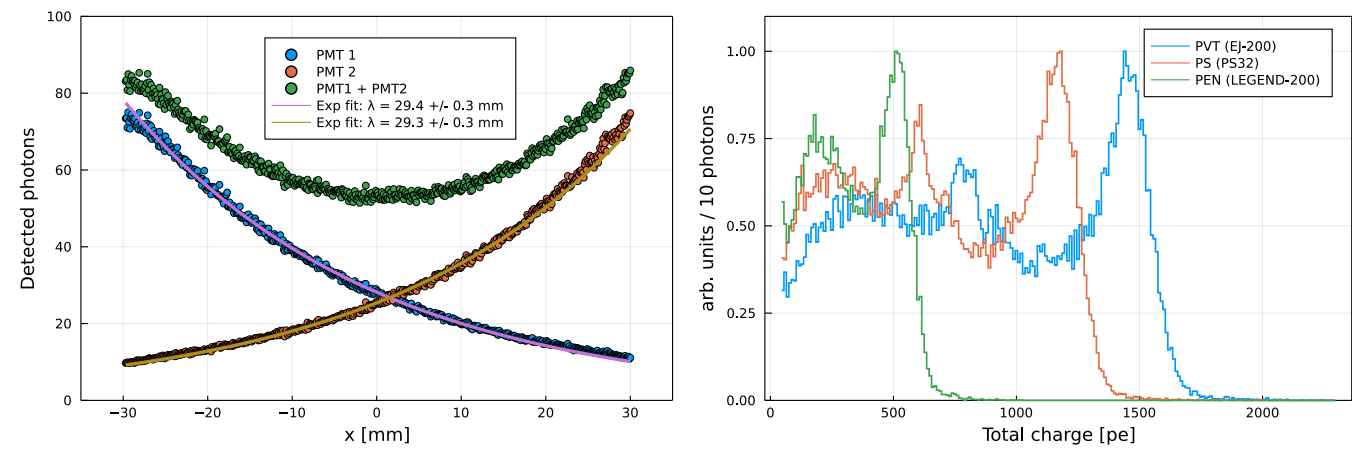

Figure 2: Left: PEN light attenuation. PEN samples of $74 \times 20 \times 1.5 \mathrm{~mm}^{3}$ were coupled to two PMTs, one in each end. As reference point it was taken the center of the sample, each PMT being placed at $\pm 37 \mathrm{~mm}$. Right: PEN light output. Samples of $30 \times 30 \times 1.7 \mathrm{~mm}^{3}$ were coupled to five PMTs. The light output is computed adding the charge of all PMTs.

\section{Conclusions}

PEN is a plastic scintillator with wavelength shifting capabilities that can be used as active structural material in low background experiments. PEN holders with excellent radiopurity and optimized geometry for usage in the LEGEND-200 experiment have been produced by injection molding. Several setups are being used to measure and characterize PEN optical properties of the LEGEND-200 production. The PEN emission spectrum, light output, attenuation and bulk absorption length have been studied. It was found a PEN light output of around 30\% of standard plastic scintillators, while a bulk absorption of around $45 \mathrm{~mm}$ at $450 \mathrm{~nm}$ has been measured.

\section{References}

[1] M. Agostini et al. [GERDA], "Final Results of GERDA on the Search for Neutrinoless Double$\beta$ Decay,” Phys. Rev. Lett. 125 (2020) no.25, 252502 doi:10.1103/PhysRevLett.125.252502 [arXiv:2009.06079].

[2] Y. Efremenko, et al. "Use of poly(ethylene naphthalate) as a self-vetoing structural material," JINST 14 (2019) no.07, P07006 doi:10.1088/1748-0221/14/07/P07006 [arXiv:1901.03579].

[3] M. Kuźniak, B. Broerman, T. Pollmann and G. R. Araujo, "Polyethylene naphthalate film as a wavelength shifter in liquid argon detectors," Eur. Phys. J. C 79 (2019) no.4, 291 doi:10.1140/epjc/s10052-019-6810-8 [arXiv:1806.04020].

[4] I. Abt, et al. "Usage of PEN as self-vetoing structural material in low background experiments," PoS ICHEP2020 (2021), 163 doi:10.22323/1.390.0163 [arXiv:2011.08983 ]. 